\title{
Erratum: Binary Black Hole Mergers in the First Advanced LIGO Observing Run [Phys. Rev. X 6, 041015 (2016)]
}

\author{
B. P. Abbott et al. \\ (LIGO Scientific and Virgo Collaborations) $)^{*, \dagger,+}$ \\ (Received 1 August 2018; published 18 September 2018)
}

DOI: 10.1103/PhysRevX.8.039903

We report an error found in the implementation of the code of the LIGO Scientific and Virgo Collaborations (LVC) as used in gravitational-wave-based estimations of possible deviations from the post-Newtonian (PN) terms expected in general relativity. The error concerned the $0.5 \mathrm{PN}$ term and affected the results previously published for GW150914 [1] in Ref. [2], for GW151226 [3] in [4], and for GW170104 in Ref. [5]. We corrected the error and present the reproduced results here, as well as in the respective Refs. [6,7]. The main conclusion, that the results are consistent with general relativity, remains.

The test for the parametrized post-Newtonian [8] deviations from the expected GR values relied on creating non-GR waveforms [2,9-13] and using them as potential matches for the observed waveforms [14-17]. In these waveforms, implemented in the frequency domain, freedom was introduced by allowing the phase coefficients describing different powers of the post-Newtonian parameter (equivalently, powers of the frequency) to assume a range of values not only the particular values prescribed by GR.

However, a coding bug was introduced, identically zeroing the deviations at $0.5 \mathrm{PN}$ in the inspiral regime (as in GR). Hence, the 0.5 PN deviations were absent in the phasing formula, though not in the junction conditions that relate the inspiral regime to the intermediate regime. Any constraints obtained in Refs. [2,4,5] only resulted from the latter.

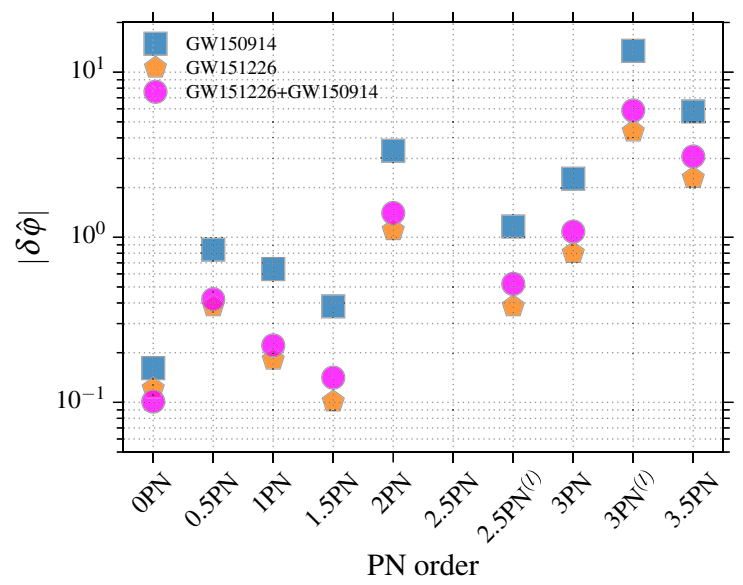

FIG. 1. This is the corrected Fig. 8 of [4], showing the $90 \%$ credible upper bounds on deviations in the PN coefficients from GW150914, from GW151226 and from both. It also fixes a previous plotting error for the 0 PN individual bounds (the GW150914 and GW151226 bounds were incorrectly drawn on top of eachother).

\footnotetext{
${ }^{*}$ Full author list given at the end of the original Letter.

†'lsc-spokesperson@ligo.org

*Virgo-spokesperson@ego-gw.it
}

Published by the American Physical Society under the terms of the Creative Commons Attribution 3.0 License. Further distribution of this work must maintain attribution to the author(s) and the published articles title, journal citation, and DOI. 
GW150914
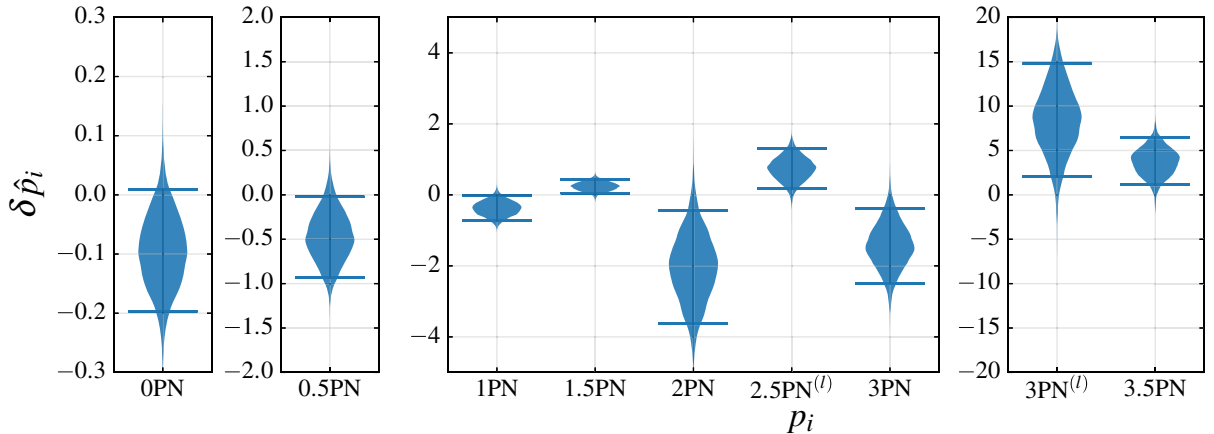

GW151226
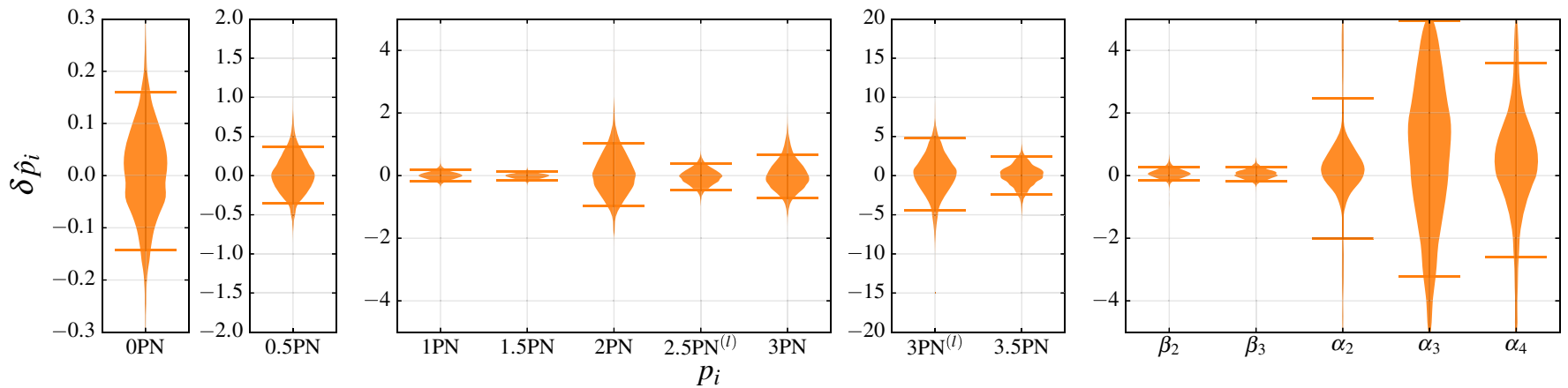

GW150914+GW151226
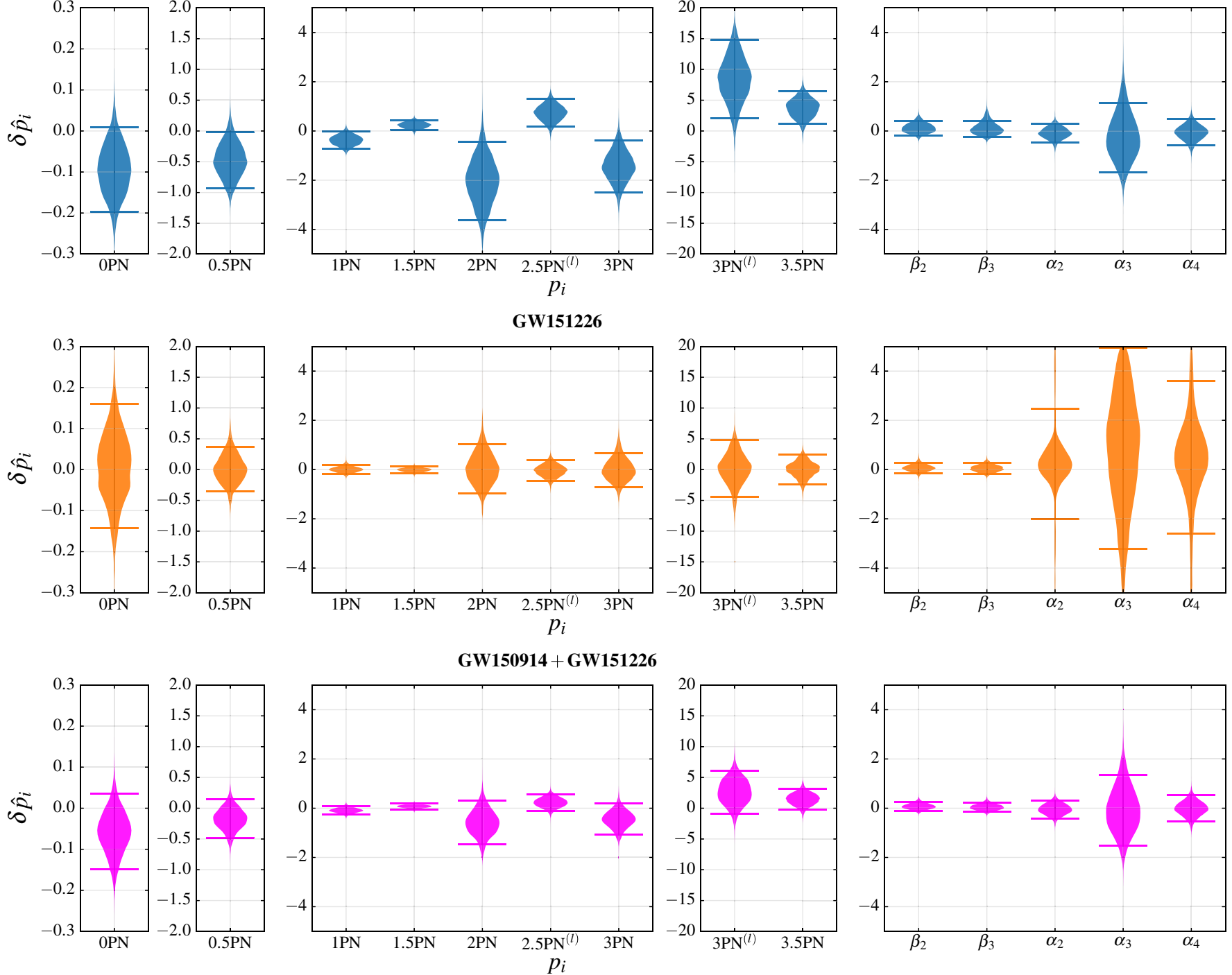

FIG. 2. This is the corrected Fig. 7 of [4], showing the posterior density distributions and $90 \%$ credible intervals for the relative deviations PN parameters $p_{i}$, as well as intermediate parameters $\beta_{i}$ and merger-ringdown parameters $\alpha_{i}$. The corrected posterior for the $0.5 \mathrm{PN}$ single-parameter analysis peaks below 0 , while previously reported to peak above; the posterior is still consistent with GR.

This error affected the results of the non-GR parameter estimation (PE) [14] pipeline tests performed for finding bounds on possible PN deviations from GR. In particular, they affected the bounds on the single deviations in the 0.5 PN term, and on the tests with multiple deviations together. These erroneous results appeared in Figs. 6 and 7 and Table I of Ref. [2], in Figs. 7 and 8 of [4], and in Fig. 9 of the Supplemental Material of Ref. [5]. The corrected versions of all of these results have been produced. The corrections for Figs. 7 and 8 of [4] appear below, while the others are available in Refs. [6,7]. All of these results are consistent with GR.

The error, introduced by erroneous caching during the optimization of the waveform generation for efficient PE, has been corrected in commit 980b3f788a445d135390eba8861b03f70133cd47 of the LALSuite [18] code. No subsequent LVC papers have been affected.

Note that, while this error also affected the analysis of GW170608 [19], the reported results require no changes: With the corrected analysis, the GR-predicted PN coefficient values continue to be consistent with the data. No change is required regarding the preliminary results reported for GW170814 [20] either.

[1] B. P. Abbott et al. (LIGO Scientific and Virgo Collaborations), Observation of Gravitational Waves from a Binary Black Hole Merger, Phys. Rev. Lett. 116, 061102 (2016). 
[2] B. P. Abbott et al. (LIGO Scientific and Virgo Collaborations), Tests of General Relativity with GW150914, Phys. Rev. Lett. 116, 221101 (2016).

[3] B. P. Abbott et al. (LIGO Scientific and Virgo Collaborations), GW151226: Observation of Gravitational Waves from a 22-SolarMass Binary Black Hole Coalescence, Phys. Rev. Lett. 116, 241103 (2016).

[4] B. P. Abbott et al. (LIGO Scientific and Virgo Collaborations), Binary Black Hole Mergers in the First Advanced LIGO Observing Run, Phys. Rev. X 6, 041015 (2016).

[5] B. P. Abbott et al. (LIGO Scientific and Virgo Collaborations), GW170104: Observation of a 50-Solar-Mass Binary Black Hole Coalescence at Redshift 0.2, Phys. Rev. Lett. 118, 221101 (2017).

[6] B. P. Abbott et al. (LIGO Scientific and Virgo Collaborations), published concurrently, Erratum: Tests of General Relativity with GW150914, Phys. Rev. Lett. 121, 129902 (2018).

[7] B. P. Abbott et al. (LIGO Scientific and Virgo Collaborations), published concurrently, Erratum: GW170104: Observation of a 50-Solar-Mass Binary Black Hole Coalescence at Redshift 0.2, Phys. Rev. Lett. 121, 129901 (2018).

[8] L. Blanchet, Gravitational Radiation from Post-Newtonian Sources and Inspiralling Compact Binaries, Living Rev. Relativity 17, 2 (2014).

[9] C. K. Mishra, K. G. Arun, B. R. Iyer, and B. S. Sathyaprakash, Parametrized Tests of Post-Newtonian Theory Using Advanced LIGO and Einstein Telescope, Phys. Rev. D 82, 064010 (2010).

[10] M. Agathos, W. Del Pozzo, T. G. F. Li, C. Van Den Broeck, J. Veitch, and S. Vitale, TIGER: A Data Analysis Pipeline for Testing the Strong-Field Dynamics of General Relativity with Gravitational Wave Signals from Coalescing Compact Binaries, Phys. Rev. D 89, 082001 (2014).

[11] L. Sampson, N. Cornish, and N. Yunes, Gravitational Wave Tests of Strong Field General Relativity with Binary Inspirals: Realistic Injections and Optimal Model Selection, Phys. Rev. D 87, 102001 (2013).

[12] T. G. F. Li, W. Del Pozzo, S. Vitale, C. Van Den Broeck, M. Agathos, J. Veitch, K. Grover, T. Sidery, R. Sturani, and A. Vecchio, Towards a Generic Test of the Strong Field Dynamics of General Relativity Using Compact Binary Coalescence, Phys. Rev. D 85, 082003 (2012).

[13] T. G. F. Li, W. Del Pozzo, S. Vitale, C. Van Den Broeck, M. Agathos, J. Veitch, K. Grover, T. Sidery, R. Sturani, and A. Vecchio, Towards a Generic Test of the Strong Field Dynamics of General Relativity Using Compact Binary Coalescence: Further Investigations, J. Phys. Conf. Ser. 363, 012028 (2012).

[14] B. P. Abbott et al. (LIGO Scientific and Virgo Collaborations), Properties of the Binary Black Hole Merger GW150914, Phys. Rev. Lett. 116, 241102 (2016).

[15] A. B. Nielsen, Compact Binary Coalescence Parameter Estimations for 2.5 Post-Newtonian Aligned Spinning Waveforms, Classical Quantum Gravity 30, 075023 (2013).

[16] J. Veitch et al., Parameter Estimation for Compact Binaries with Ground-Based Gravitational-Wave Observations Using the LALInference Software Library, Phys. Rev. D 91, 042003 (2015).

[17] S. Vitale, Multiband Gravitational-Wave Astronomy: Parameter Estimation and Tests of General Relativity with Space- and Ground-Based Detectors, Phys. Rev. Lett. 117, 051102 (2016).

[18] LSC Algorithm Library Suite, https://wiki.ligo.org/DASWG/LALSuite, https://git.ligo.org/lscsoft/lalsuite.

[19] B. P. Abbott et al. (LIGO Scientific and Virgo Collaborations), GW170608: Observation of a 19-Solar-Mass Binary Black Hole Coalescence, Astrophys. J. 851, L35 (2017).

[20] B. P. Abbott et al. (LIGO Scientific and Virgo Collaborations), GW170814: A Three-Detector Observation of Gravitational Waves from a Binary Black Hole Coalescence, Phys. Rev. Lett. 119, 141101 (2017). 